\title{
Nonlinear Pulse Reshaping in Optical Fibers
}

\author{
S. O. Iakushev' ${ }^{1}$ I. A. Sukhoivanov ${ }^{2}$, O. V. Shulika ${ }^{1}$, \\ J. A. Andrade-Lucio ${ }^{2}$ and A.G. Perez ${ }^{2}$ \\ ${ }^{1}$ Kharkov National University of Radio Electronics \\ ${ }^{2}$ DICIS, University of Guanajuato \\ ${ }^{1}$ Ukraine \\ ${ }^{2}$ Mexico
}

\section{Introduction}

The propagation of ultrashort laser pulses in the optical fibers is connected with a plenty of interesting and practically important phenomena. Unique dispersive and nonlinear properties of the optical fibers lead to various scenarios of the pulse evolution which are resulted in particular changes of the pulse shape, spectrum and chirp. The modern age of the optical fibers starts from the 1960s with the appearance of the first lasers. These fibers were extremely lossy but new suggestion on the geometry with the single mode operation (Kao et al., 1966) which was obtained by theoretical calculations based on the Maxwell's equations, and the development of a new manufacturing process (French et al., 1974) have led to the achievement of the theoretical minimum of the loss value $0.2 \mathrm{~dB} / \mathrm{m}$ (Miya et al., 1979). The investigations of the nonlinear phenomena in the optical fibers have been continuously gained by the decreasing loss. Loss reduction in the fibers made possible the observation of such nonlinear processes which required longer propagation path length at the available power levels in the 1970s. Stimulated Raman Scattering (SRS) and Brillouin scattering (SBS) were studied first (Ippen et al., 1972). Optical Kerr-effect (Stolen et al., 1973), parametric four-wave mixing (FWM) (Stolen et al., 1974) and self-phase modulation (SPM) (Stolen et al., 1978) were observed later. The theoretical prediction of the optical solitons as an interplay of the fiber dispersion and the fiber nonlinearity was done as early as 1973 (Hasegawa et al., 1973) and the soliton propagation was demonstrated seven years later in a single mode optical fiber (Mollenauer et al., 1980).

Discovery of the optical solitons have revolutionized the field of the optical fiber communications. Nowadays solitons are used as the information carrying "bits" in optical fibers (Hasegawa et al., 2003). This is resulted from unique properties of the solitons. In general, the temporal and spectral shape of a short optical pulse changes during propagation in a medium due to the self-phase modulation and chromatic dispersion. This actually limits the transmission bit rate in optical fibers. Under certain circumstances, however, the SPM and dispersion can exactly cancel each other producing a self-localized waveform called the solitary wave. Due to the particle-like nature of these solitary waves during mutual interactions they were called solitons. Solitons are formed when GVD is 
anomalous, and also, the pulse energy and duration have to meet a particular condition in order to provide the formation of a stable soliton called the fundamental soliton. Fundamental solitons preserve their temporal and spectral shape even over long propagation distances. However, if the pulse energy is larger, e.g. the square of an integer number times the fundamental soliton energy, then a higher-order soliton is formed. Such pulses do not have a preserved shape, but their shape varies periodically.

Solitons possess a number of important features. An optical soliton is a solution of the nonlinear Schrödinger equation (NLSE) in the form of secant-shaped pulse (Zakharov et al., 1972). This soliton solution is very stable: even for substantial deviations of the initial pulse from the exact secant shape, the pulse tends to correct his shape towards the correct soliton form. It means that initial Gaussian or super-Gaussian pulse transforms to the soliton after some propagation distance in a fiber. Solitons are also very stable against changes of the properties of the medium, provided that these changes occur over distances which are long compared with a soliton period. It means that solitons can adapt their shape to slowly varying parameters of the medium.

Owing to the features mentioned above the solitons play important role not only in optical fiber communications but also in ultrashort lasers as well. Soliton mode locking is a frequently used technique for producing high-quality ultrashort pulses in the bulk and fiber lasers (Brabec et al., 1991; Duling III, 1991). Solitons have been the subject of intense theoretical and experimental studies in many different fields, including hydrodynamics, nonlinear optics, plasma physics, and biology (Gu, 1995; Akhmediev et al., 2008). Up to date many other kinds of solitons have been discovered depending on the dispersive and nonlinear properties of the fibers. In the context of the optical solitons, one can distinguish temporal or spatial solitons, depending on whether the confinement of light occurs in time or space. Temporal solitons have been mentioned above, they represent optical pulses which maintain their shape, whereas spatial solitons represent self-guided beams that remain confined in the transverse directions orthogonal to the direction of propagation. A spatial soliton arises when the self-focusing of an optical beam balances its natural diffractioninduced spreading. Spatiotemporal optical solitons can demonstrate both temporal and spatial localization of light (hence the term "light bullets"), which are nondiffracting and nondispersing wavepackets propagating in a nonlinear optical media. Other types of solitons include: dispersion-managed solitons, dissipative solitons, dark solitons, Bragg solitons, vector solitons, vortex solitons (Kivshar et al., 2003).

In spite of unique properties of the optical solitons the difficulties arise with generation of the high power pulses and their delivering through the optical fibers due to distortions and break-up effects. Particularly, the fundamental soliton exists at only one particular power level and propagation of higher-power pulses excites the higher-order solitons which are sensitive to perturbation and break-up through the soliton fission (Kodama et al., 1987; Dudley et al., 2006). Whereas in the normal dispersion regime the pulse propagation is subject to instability through the appearance of the optical wave breaking (OWB) (Tomlinson et al., 1985; Anderson et al., 1992). It was shown that optical intensity shock formation precedes the OWB appearance (Rothenberg, 1989). The shock occurs when the more intense parts of the pulse, owing to the nonlinearity, are travelling at the speeds which are different from those of the weaker parts. This reshaping evolves to a breaking singularity, when the top of the shock actually overtakes its bottom, resulting in a region of multivalued solutions. 
Break-up effects in general restrict pulse propagation in an optical fiber, especially concerning the high-power pulses. However, in 1990s it was theoretically predicted that pulses with parabolic intensity profile and a linear frequency chirp can propagate without wave breaking in the normal dispersive optical fiber (Anderson et al., 1993). Then it was shown numerically that ultrashort pulses injected into a normal dispersion optical fiber amplifier evolve towards the parabolic pulse profile with amplification and, moreover, retained their parabolic shape even as they continued to be amplified to higher powers (Tamura et al., 1996). In 2000 an experimental observation of parabolic pulse generation in the fiber amplifier with normal dispersion applying advanced ultrashort pulse measurement technique of the frequency-resolved optical gating (FROG) was reported (Fermann et al., 2000).

This novel class of the optical pulses exhibits several fundamental features. Parabolic pulses propagate in a self-similar manner, holding certain relations (scaling) between the pulse power, width, and chirp parameter. The scaling of the amplitude and width of both the temporal amplitude and spectrum depends only on the amplifier parameters and the input pulse energy, and is completely independent of the input pulse shape. Moreover, the pulse chirp is completely independent of the propagation distance. A major result of the theoretical analysis becomes the demonstration that the self-similar parabolic pulse is an asymptotic solution to the NLSE with gain, representing a type of nonlinear "attractor" towards which any arbitrarily-shaped input pulse of the given energy would converge with sufficient distance (Fermann et al., 2000; Kruglov et al., 2002). Thus, initial arbitrary shaped pulse propagating in a normally dispersive fiber amplifier reshapes itself into a pulse having a parabolic intensity profile combined with a perfectly linear chirp. Theoretical and numerical results obtained later have shown that the self-similar parabolic pulses shape is structurally stable (Kruglov et al., 2003).

Self-similarity is a fundamental property of many physical systems and has been studied extensively in diverse areas of physics such as hydrodynamics, mechanics, solid-state physics and theory of elasticity (Barenblatt, 1996). Discovery of the self-similar pulse propagation in the optical fibers attracts much interest not only from theoretical standpoint but due to possible practical applications as well (Dudley et al., 2007). By analogy with the well-known stable dynamics of the solitary waves - solitons, these self-similar parabolic pulses have been termed as similaritons. One has to note that solitons themselves can also be interpreted as an example of self-similarity (Barenblatt, 1996). In contrast to the optical solitons however, the similaritons in optical fibers can tolerate strong nonlinearity without wave breaking. The normal GVD effectively linearizes the accumulated phase of the pulse allowing for the spectral bandwidth to increase without destabilizing the pulse. Solitons maintain their shape, width, and amplitude, whereas similaritons maintain their shape but not their width or amplitude; instead of that the certain relations between pulse power, width, and chirp parameter are hold.

Application of similaritons provided a significant progress in the field of pulse generation and amplification in fiber systems. The remarkable properties of the parabolic pulses have been applied to the development of a new generation of high-power optical fiber amplifier (Schreiber et al., 2006). Self-similar propagation regime allows avoiding the catastrophic pulse break up due to excessive nonlinearity. However, in contrast to the well-known chirped pulse amplification method, where the aim is to avoid 
nonlinearity by dispersive pre-stretching before amplification, a self-similar amplifier actively exploits the nonlinearity. This allows obtaining output pulses that are actually shorter than after recompression of the initial input pulse. Similariton approach has been applied also to the development of high-power fiber lasers ("similariton laser") allowing overcome existing power limitations of soliton modelocking (Ilday et al., 2004). Parabolic pulses are of great interest for a number of applications including, amongst others, the highly coherent continuum sources development and optical signal processing (Finot et al., 2009).

Parabolic pulses are generated usually in the active fiber systems such as amplifiers or lasers. However, last time it is also of interest to study alternative methods of generating parabolic pulses, especially in the context of non-amplification usage, such as optical telecommunications. Some approaches were proposed for the generation of parabolic pulses in the passive fiber systems, such as dispersion decreasing fibers (Hirooka et al., 2004) and fiber Bragg gratings (Parmigiani et al., 2006). Then it was found that nonlinearity and normal dispersion in the simple passive fiber can provide pulse reshaping towards the parabolic pulse at the propagation distance preceding the optical wave breaking (Finot et al., 2007). But with longer propagation distance the pulse shape doesn't remain parabolic. However, recently the possibility to obtain nonlinear-dispersive similaritons in the passive fibers at longer propagation distances was demonstrated (Zeytunyan et al., 2009; Zeytunyan et al., 2010). In this regime pulse propagates actually in some steady-state mode when pulse shape and spectrum does not change significantly with propagation distance and they repeat each other due to spectronic nature and moreover the chirp of such pulse is linear. Thus, there is some analogy with parabolic pulse formation in fiber with gain. However, the shape of the pulses obtained in passive fiber is different from the fully parabolic one. Under some special initial conditions even triangular pulses can be obtained (Wang et al., 2010).

Based on our recent results (Sukhoivanov et al., 2010; Yakushev et al., 2010) here we will discuss nonlinear pulse transformations in the normal dispersive regime of the passive fibers. Particularly in the weak nonlinear case (soliton order is less than 10) we will present numerical results regarding the pulse shape evolution towards parabolic profile depending on the initial pulse shape, soliton number and fiber length. We will show that some quasiparabolic pulses with linear chirp can be obtained. The influence of the initial pulse parameters and fiber parameters on the shape of quasi-parabolic pulses will be investigated. We also reveal the role of the third-order dispersion.

In the strong nonlinear case (soliton order is larger than 10) optical wave breaking becomes significant. Previously, it was shown that oscillations induced by OWB are vanishing at longer distances and pulse transforms to the chirped trapezium-shaped pulse (Karlsson, 1994). However, there is no proper explanation of this phenomenon and further pulse evolution has not been investigated. We will present numerical results concerning to the pulse transformations at the much longer distance, until steady-stage regime is achieved. At this stage the pulse shape becomes nearly smooth with a linear chirp. We explain the OWB cancellation by the action of normal dispersion, which tends to flatten nonlinear chirp, induced by self-phase modulation during the pulse propagation in a fiber. It is possible to obtain the resulted parabolic pulse profile or a triangular one in the steady-state regime depending on the initial conditions. 


\section{Theoretical model}

The evolution of an ultrashort pulse during its propagation in a normal-dispersion fiber with Kerr nonlinearity is well described by the nonlinear Schrödinger equation (NLSE) (Agrawal, 2007):

$$
\frac{\partial A}{\partial z}=-\frac{\alpha}{2} A-\frac{i \beta_{2}}{2} \frac{\partial^{2} A}{\partial T^{2}}+\frac{\beta_{3}}{6} \frac{\partial^{3} A}{\partial T^{3}}+i \gamma|A|^{2} A,
$$

where $A(z, t)$ is the slowly varying complex envelope of the pulse; $\alpha$ is the loss; $\beta_{2}$ is the second order dispersion; $\beta_{3}$ is the third order dispersion; $\gamma$ is the nonlinear coefficient; $T$ is the time in a co-propagating time-frame; $z$ is the propagation distance. The formation of the nearly parabolic pulses in the passive optical fibers is basically governed by the interplay of normal dispersion and nonlinearity. But here we extend standard NLSE by including the fiber loss and the third-order dispersion in order to investigate the impact of these factors on the parabolic pulse formation. Equation (1) is solved numerically using the split-step Fourier method (Agrawal, 2007). For the subsequent discussion it is convenient to use following notations for the dispersion length $L_{D}$, dispersion length associated with the third-order dispersion $L_{D}^{\prime}$, nonlinear length $L_{N L}$, soliton order $N$ and normalized length $\xi$ :

$$
L_{D}=T_{0}^{2} /\left|\beta_{2}\right|, L_{D}^{\prime}=T_{0}^{3} /\left|\beta_{3}\right|, L_{N L}=1 /\left(\gamma P_{0}\right), N=\sqrt{L_{D} / L_{N L}}, \quad \xi=z / L_{D} .
$$

here $P_{0}$ is the initial pulse peak power, $T_{0}$ is the initial pulse duration (half-width at $1 / \mathrm{e}-$ intensity level). At first, we investigate the weak nonlinear case when $1<N<10$. In this case the self-phase modulation (SPM) dominates over the group velocity dispersion (GVD) during the initial stages of the pulse evolution, then pulse evolution preliminary governed by GVD. Larger value of $N>10$ leads to the stronger impact of the nonlinearity, leading to the optical wave breaking phenomenon.

In the following the quality of the pulse is estimated through the deviation of its temporal/spectral intensity profile $|A(T)|^{2}$ and a parabolic fit $\left|A_{p}(T)\right|^{2}$ of the same energy (Finot et al., 2006), which is expressed as the misfit parameter $M$ :

$$
M^{2}=\frac{\int\left(|A|^{2}-\left|A_{p}\right|^{2}\right)^{2} d \tau}{\int|A|^{4} d \tau} .
$$

The expression for a parabolic pulse of energy $U_{p}=4 P_{p} T_{p} / 3 \sqrt{2}$ is given by:

$$
\begin{cases}A_{p}(T)=\sqrt{P_{p}} \sqrt{1-2 T^{2} / T_{p}{ }^{2},} & |T| \leq T_{p} / \sqrt{2} \\ A_{p}(T)=0, & |T|>T_{p} / \sqrt{2}\end{cases}
$$

where $P_{p}$ is the peak power of the parabolic pulse, $T_{p}$ is the duration of the parabolic pulse (full-width at half-maximum). The misfit parameter $M$ allows estimating the pulse shape 
imperfection as compared to the parabolic shape; the smaller value of $M$ shows better fit to the parabolic waveform. Usually we can consider a pulse shape to be close enough to the parabolic one when $M<0.04$.

\section{Pulse reshaping in the weak nonlinear case}

\subsection{The influence of initial pulse shape and chirp on the resulted pulse profile}

At first, we address pulse reshaping in the weak nonlinear case $1<N<10$ at the normal dispersive optical fiber. Basically pulse reshaping is governed by the interplay between SPM and GVD. One can separate two stages of the pulse evolution. In the first stage within the short propagation distance the SPM effect is strong. Owing to that the chirp becomes nonlinear and fast reshaping of the pulse temporal and spectral shapes occurs. It was shown that a parabolic pulse can be obtained from initial unparabolic pulse at this stage at the particular propagation distance (Finot et al., 2007). However, further pulse reshaping continues and pulse shape doesn't remain parabolic. Finally, SPM action goes down and GVD dominates over the longer propagation distance. At this stage pulse propagates in the nearly linear propagation regime. Here pulse chirp becomes linear and pulse temporal and spectral shape changes slowly and actually maintains some particular profile. However, the resulted pulse shape strongly differs from the initial pulse shape (Zeytunyan et al., 2009). Here, we investigate comprehensively pulse reshaping depending on the initial pulse parameters and fiber parameters.

Firstly, we investigate pulse reshaping in the case of initial Gaussian pulse shape. The Mmap presented in Fig. 1 (a) demonstrates the dependence of the misfit parameter $M$ on the soliton order $N$ and normalized length $\xi$. We also calculated the M-plot shown in Fig. 1 (b), which represents the dependence of the misfit parameter $M$ versus $\xi$ for a particular value $N$. Both the deviation of the temporal pulse shape and the deviation of the spectrum shape from the parabolic one were calculated. In the last case equations (3)-(4) are applied for the spectral pulse amplitude.

On Fig. 1(a) three specific areas are denoted by numbers. Number 1 indicates narrow and dip vertical area within the short propagation distance $(\xi<1)$. This area has been extensively investigated by Finot et al (Finot et al., 2007) and Boscolo et al (Boscolo et al., 2008). It is possible to achieve here fully parabolic pulse $(M \sim 0.04)$. However, a suitable $M$ is achieved only over the narrow range of propagation distances, then pulse shape changes. Moreover, from Fig. 1 (b) we can see that only temporal pulse profile achieves parabolic shape, whereas the spectral shape shows maximal deviation from the parabolic shape here. We should point out here also an adjacent wider vertical area (Number 2). Here the propagation distance is also small $(1<\xi<2)$ and misfit parameter $M$ is close to that one in the first area.

Now let us look at the pulse reshaping within the longer propagation distance. We are especially interested in this region because some steady-state regime is achieved here. This implies that pulse shape and spectrum do not change sufficiently with propagation distance any more. From Fig. 1(b) we can conclude that steady-state starts approximately from $\xi>5$. Here both temporal and spectral curves are reduced very slowly and they are very close to each other. The value of $N$ affects strongly on the misfit parameter $M$ in the steady-state. From Fig. 1 (a) one can see that $M$ sufficiently increases with increasing of $N$. It means that 
pulse shape in the steady-state will be different depending on the $N$. In Fig.1(a) we can point out also the horizontal valley $1.5<N<2.5$, where misfit parameter achieves smaller value as compared to the outside value of $N$ (Number 3). In this area one can achieve $M \sim 0.07$.

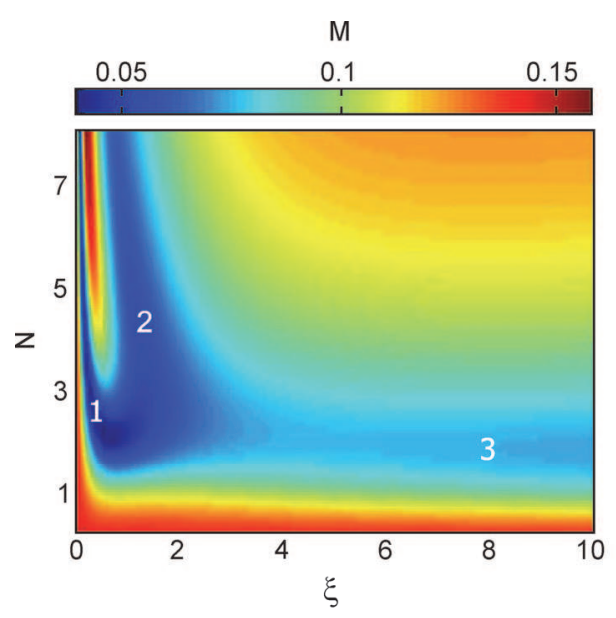

a)

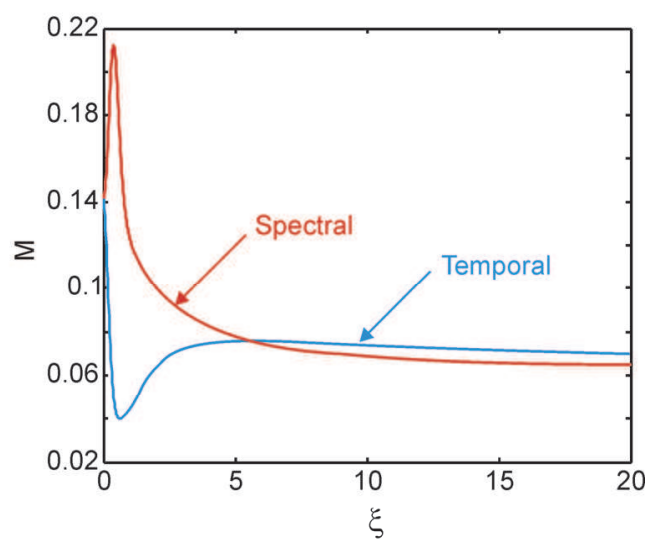

b)

Fig. 1. a) Evolution of the misfit parameter $M$ versus $N$ and $\xi$ in the case of initial Gaussian pulse shape. Numbers at the figure denote three specific areas described in the text. b) Evolution of the misfit parameter $M$ versus $\xi$ for the temporal and spectral pulse shapes $(N=2)$.

Now we investigate pulse reshaping in the case of initial secant pulse shape. The M-map for the secant pulse shape is shown in Fig. 2 (a) and appropriate M-plot is shown in Fig. 2 (b). From Fig. 2 we see that pulse reshaping process in the case of initial secant pulse is different from that one for Gaussian. Within the short propagation distance we find only one area where pulse achieves the parabolic shape. Moreover one can see that steady-state regime arises at much longer propagation distance as compared to the Gaussian. We find the same horizontal valley $1.5<N<2.5$, where misfit parameter achieves smaller value. However, here the resulted pulse shape in the steady-state differs stronger from the parabolic one as compared to the previous Gaussian pulse.

Now we investigate pulse reshaping in the case of initial super-Gaussian (2-nd order) pulse shape. The M-map for super-Gaussian pulse shape is shown in Fig. 3 (a) and corresponding M-plot is shown in Fig. 3 (b).

From Fig. 3 we can see that pulse reshaping process in the case of initial super-Gaussian pulse inevitably leads to the fully parabolic pulse for $N>1$. Steady-state regime arises very fast as compared to the previously investigated initial pulse shapes. However, one should note that in the case of pulses with steeper leading and trailing edges (higher order superGaussian pulses) the resulted pulse profile in the steady-state regime differs stronger from the parabolic one. 


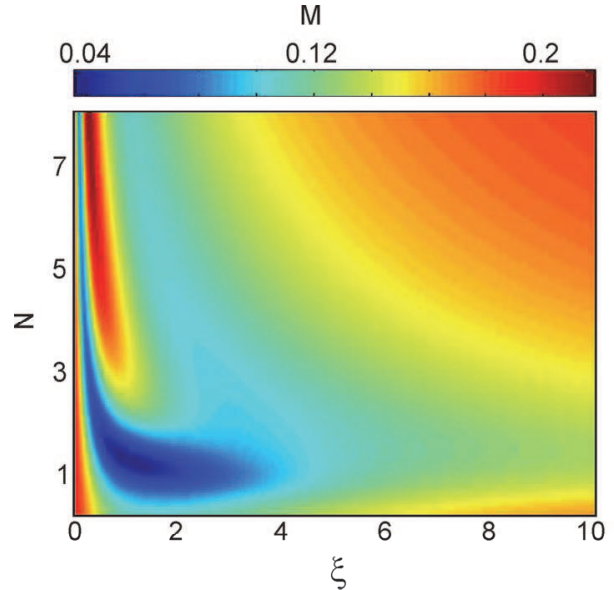

a)

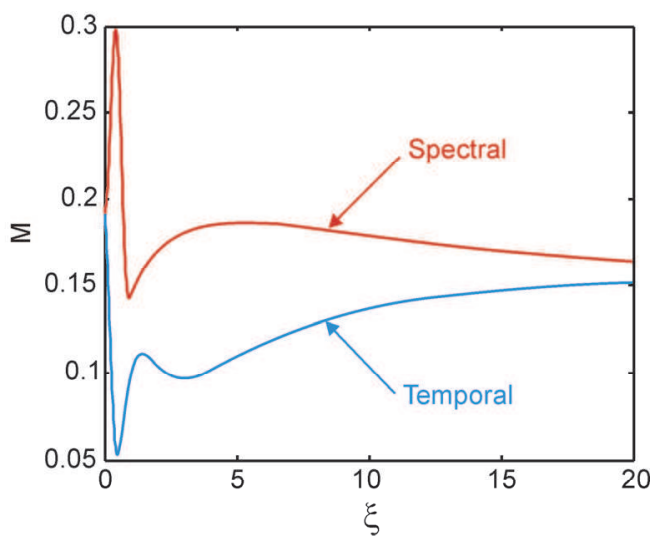

b)

Fig. 2. a) Evolution of the misfit parameter $M$ versus $N$ and $\xi$ in the case of initial secant pulse shape. b) Evolution of the misfit parameter $M$ versus $\xi$ for the temporal and spectral pulse shapes $(N=2)$.
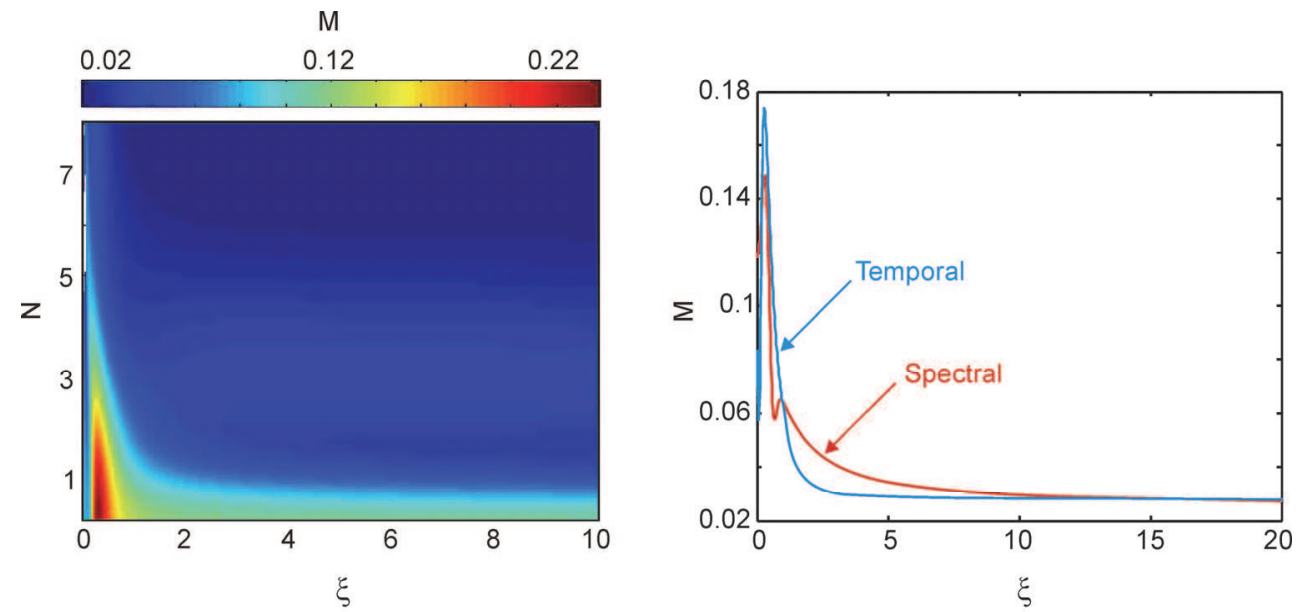

Fig. 3. a) Evolution of the misfit parameter $M$ versus $N$ and $\xi$ in the case of initial superGaussian pulse shape. b) Evolution of the misfit parameter $M$ versus $\xi$ for the temporal and spectral pulse shapes $(N=2)$.

Results presented above were obtained in the case of initially unchirped pulses, now we examine the influence of the initial pulse chirp on the resulted pulse shape in the steadystate regime. We include chirp in the initial pulse in the following way:

$$
A_{\text {chirp }}=A_{\text {unchirp }} \exp \left(i C \frac{T^{2}}{2 T_{0}^{2}}\right),
$$


where $A_{\text {unchirp }}$ is the waveform of an unchirped initial pulse; $C$ - chirp parameter.

This leads to the initial linear dependence of instantaneous frequency:

$$
\omega=\omega_{0}+C \frac{T}{T_{0}^{2}}
$$

where $\omega_{0}$ is a central frequency of the pulse spectrum. The instantaneous frequency increases linearly from the leading to the trailing edge of the pulse for $C>0$ (positive chirp) while the opposite process occurs for $C<0$ (negative chirp). We compare pulse evolution for initial Gaussian pulse with positive chirp, negative chirp and unchirped $(C=0)$, Fig. 4.

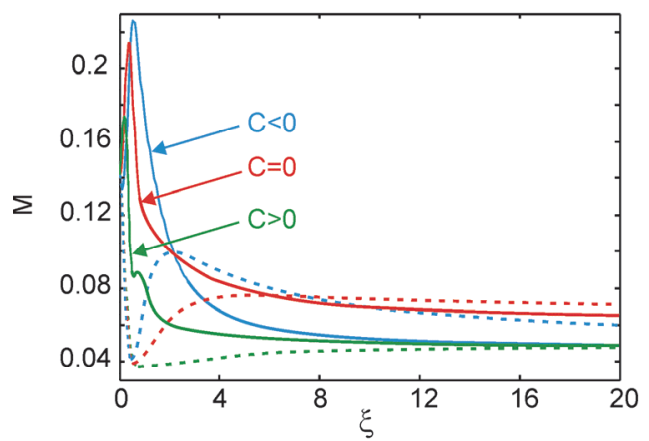

a)

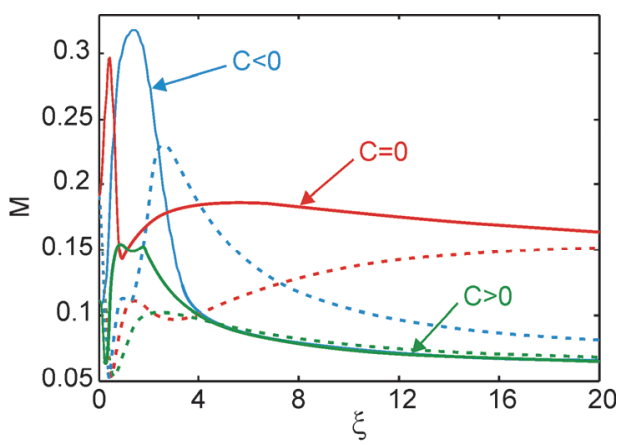

b)

Fig. 4. Evolution of the misfit parameter $M$ versus $\xi$ for the temporal and spectral pulse shapes $(N=2)$ in the case of initial pulse with initial positive chirp, negative and unchirped. The absolute value of initial chirp parameter here is 0.5. a) - initial Gaussian pulse; b) - initial secant pulse. Dotted lines denote temporal curves; solid lines - spectral curves.

From Fig. 4 we can see that initial chirp is able to change significantly pulse evolution. We can see that the maxima of the spectral and temporal curves at the short propagation distances increase when initial chirp is negative and decrease when initial chirp is positive. In the steady-state regime positive chirp leads to the lower deviation from the parabolic shape both for temporal and spectral profiles and steady-state regime arises faster. In the case of negative chirp the spectral shape also becomes closer to the parabolic, but the temporal curve indicates stronger deviation. So, we can conclude that application of pulses with initial positive chirp is more preferable. For initial Gaussian pulse we can obtain misfit parameter $M \sim 0.05$ and for secant pulse $M \sim 0.08$ at $\xi=8$.

In the case of initial super-Gaussian pulse the fully parabolic pulse is achieved already for unchirped pulse. Our calculations show that adding the initial chirp in this case does not change sufficiently pulse evolution presented in Fig. 3 a), there are only slight changes of the distance where steady-state regime is achieved. Increasing the magnitude of the positive chirp is able to provide actually a fully parabolic pulse from the initial Gaussian pulse. Fig. 5 shows the pulse shape, spectrum and chirp for $C=1$ and $\xi=4$. We see that both spectral and temporal profiles are parabolic $(M \sim 0.03)$ and chirp is actually linear over the whole pulse. 


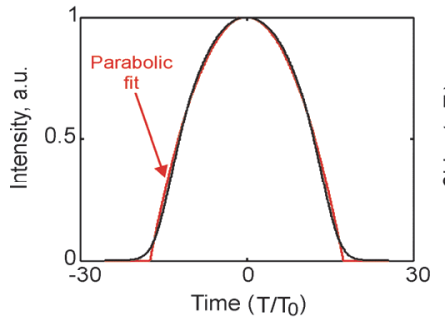

a)

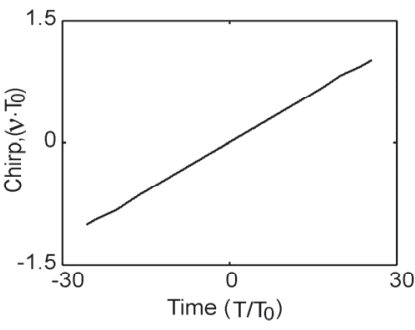

b)

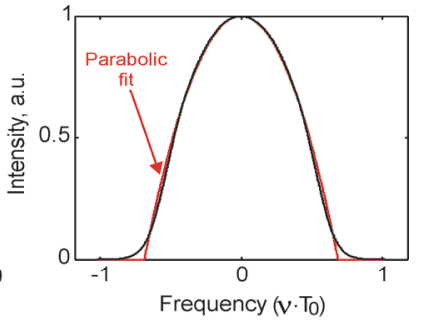

c)

Fig. 5. Normalized pulse temporal intensity - (a), spectrum - (c) and chirp (b) in the steadystate regime produced from initial Gaussian pulse with positive chirp $(N=2, \xi=4, C=1)$. Red curves show corresponding parabolic fits.

In the case of initial secant pulse a stronger initial positive chirp and longer propagation distance is required in order to achieve better quasi-parabolic pulse. For example, our calculations show that quasi-parabolic pulse with $M \sim 0.05$ for both spectral and temporal profile can be obtained when $N=2, \xi=8, C=2.5$.

\subsection{Influence of the third-order dispersion, loss and gain}

The self-phase modulation and the group velocity dispersion are main factors of pulse reshaping towards the parabolic shape in the passive optical fiber. However other factors are able to change the pulse reshaping. At first we investigate the impact of third-order dispersion (TOD) associated with the 3-rd order time derivative in the NLSE (1). In the linear regime $(\gamma=0)$ TOD effects play a significant role only if $L_{D}^{\prime} / L_{D} \leq 1$ (Agrawal, 2007). In this case TOD leads to the asymmetric broadening of the pulse with an oscillated tail near one of its edges. In the presence of nonlinearity the TOD influence becomes more complex. The impact of TOD in the steady-state regime of a passive fiber is quite similar to that one in the case of similariton pulse propagation (Latkin et al., 2007; Bale et al., 2010). From Fig. 6 we see that pulse temporal and spectrum profiles become asymmetric with the peak shifted towards one of the edges depending on the sign of the TOD; pulse chirp becomes nonlinear. Further increasing of TOD leads to the development of the optical shock-type instabilities. In the temporal profile we can observe fast and deep oscillations and a lateral satellite in the pulse spectrum arises.

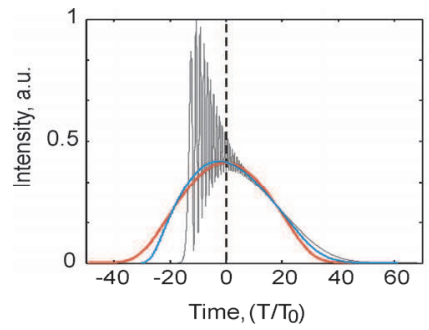

a)



b)

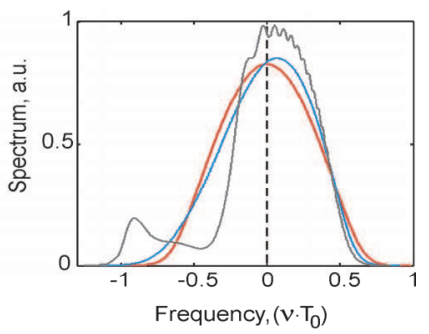

C)

Fig. 6. Pulse evolution in the steady-state under the TOD impact ( $N=2, \xi=8$ ). (a) - Pulse temporal profile. (b) - Chirp. (c) - Spectrum. Color indicates the relative magnitude of TOD: red curve - without TOD; blue curve - $L_{D}^{\prime} / L_{D}=7.3$; gray curve - $L_{D}^{\prime} / L_{D}=3.3$. 
We estimate here distortion of the pulse shape due to the TOD impact applying (3) for perturbed pulse waveform and appropriate unperturbed pulse in the steady-state (instead of parabolic fit). Fig. 7 shows evolution of misfit parameter $M$ versus $L_{D}^{\prime} / L_{D}$ for various initial pulse shapes.

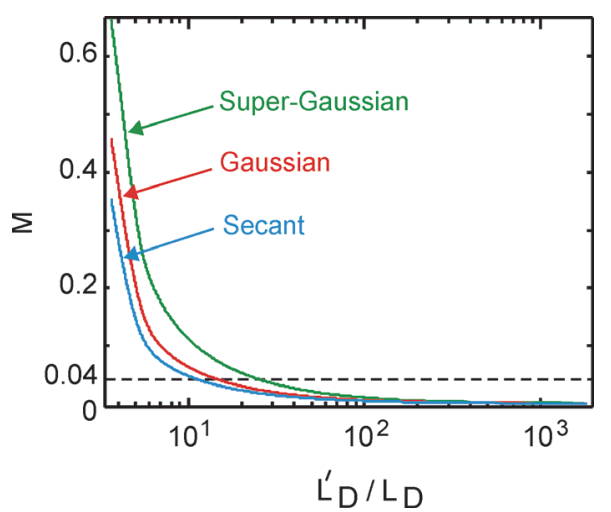

Fig. 7. Evolution of the misfit parameter $M$ versus $L_{D}^{\prime} / L_{D}$ which demonstrates TOD impact on the pulse profile $(N=2, \xi=8)$.

From Fig. 7 we see that super-Gaussian pulse is the most sensitive to the TOD, whereas secant pulse is the least sensitive. Level 0.04 in Fig. 6 indicates the border, where pulse distortions become significant. Less value of misfit parameter can be achieved when $L_{D}^{\prime} / L_{D}>30$ for all pulse shapes. This condition should be taken into account in order to select suitable fiber for pulse reshaping.

Next we investigate the impact of loss and gain on the pulse reshaping in the steady-state regime. Both effects can be taken into account using the term $\alpha$ in NLSE (1). When $\alpha>0$ it means the presence of loss in the fiber; $\alpha=0$ - lossless case; $\alpha<0$ indicates gain. Fig. 8 shows evolution of misfit parameter $M$ (given by (3)-(4)) versus $\xi$ both for the temporal and spectral pulse shapes. Initial pulse shape here is Gaussian, when initial pulse shape is secant or super-Gaussian pulse evolution is qualitatively similar. Total loss/gain was chosen to be $20 \mathrm{~dB}$, it corresponds to approximately total gain of $2.3 \mathrm{~m}$ of $\mathrm{Yb3}+$ doped fiber. Typical attenuation of the photonic crystall fibers (PCF) can exceed tens of $\mathrm{dB} / \mathrm{km}$, such that total loss $20 \mathrm{~dB}$ correspond to hundreds meters of PCF.

From Fig. 8 we can see that within the short propagation distance both loss and gain don't affect sufficiently pulse evolution, because dotted and dashed lines nearly coincide with appropriate solid lines. However with increasing the propagation distance the loss/gain influence becomes stronger. In the case of loss the dotted spectral and temporal curves are nearly horizontal and they tend asymptotically to some limit without intersection (at least within the calculated propagation distance). Actually it means that the presence of loss damps the strength of the pulse reshaping and the resulted pulse shape differs stronger from the parabolic profile. The influence of the gain is opposite to that one of the loss. Gain enhances pulse reshaping towards the parabolic shape and we obtain actually a similariton propagation regime. Spectral profile (green solid line in Fig. 8) tends to parabolic shape 
faster as compared to the temporal one. However, one should note that spectral curve doesn't achieve $M=0$, we can see only some minimum around $\xi=15$ and then this curve grows up. This behavior is related to the arising the oscillations in the pulse spectral profile in the similariton propagation regime (Kruglov et al., 2002).

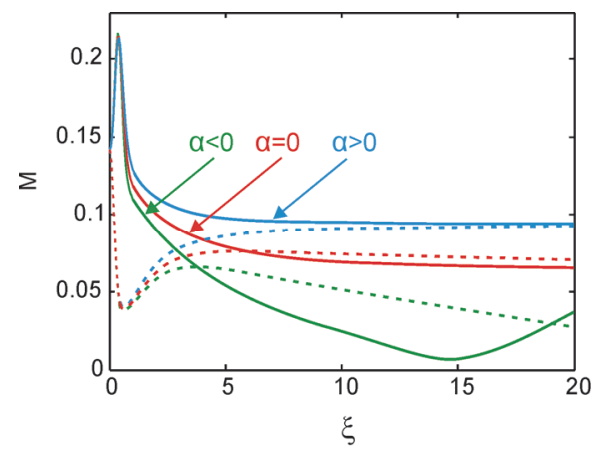

Fig. 8. Evolution of the misfit parameter $M$ versus $\xi$ for the temporal and spectral pulse shapes $(N=2)$. Blue lines denote $\alpha>0$; red lines $-\alpha=0$; green lines $-\alpha<0$. Dotted lines denote temporal curves, solid lines - spectral curves.

Based on the presented results we can estimate some practical conditions for achieving the quasi-parabolic pulses in a passive fiber. One can see that initial super-Gaussian pulse is the best choice to obtain parabolic pulses in a passive optical fiber, but state of the art ultrashort lasers generate usually secant or Gaussian waveform. In this case an initial Gaussian pulse is more preferable than secant, because the resulted pulse shape can be closer to the parabolic one. Next point is that selected fiber should provide suitable dispersion properties at the operation wavelength. Dispersion should be normal and flat enough in order to reduce the impact of high-order dispersion $\left(L_{D}^{\prime} / L_{D}>30\right)$, moreover the higher amount of secondorder dispersion allows usage of shorter pieces of fiber due to the smaller dispersion length $L_{D}$. For example, for realistic dispersion $\beta_{2}=20 \mathrm{ps}^{2} / \mathrm{km}$ and initial pulse duration $100 \mathrm{fs}$, the required fiber length $\left(8 L_{D}\right)$ is about $1.4 \mathrm{~m}$. Application of photonic crystal fibers seems attractive due to the possibility to design the desired dispersion properties by varying geometrical fiber parameters such as the lattice pitch and diameter of air-holes. Because the required fiber length is quite short the influence of attenuation on the pulse reshaping process is expected to be negligible. Next, by varying the initial pulse peak power and/or selecting fiber with appropriate nonlinear coefficient one can achieve operation within the desired range $1.5<N<2.5$, where pulse shape in the steady-state regime is closest to the parabolic one (for Gaussian pulse $M \sim 0.07$ ). Application of pulses with initial positive chirp is desirable to further decrease misfit parameter and required fiber length.

\section{Pulse reshaping in the strong nonlinear case}

\subsection{Optical wave breaking cancellation}

Now, we investigate pulse reshaping in the strong nonlinear case $(N>10)$ in the normal dispersive optical fibers. The main difference here is appearing of the optical wave breaking 
at the initial stage of the pulse propagation. The appearing of optical wave breaking has been investigated theoretically and experimentally (Tomlinson et al., 1985; Rothenberg, 1989; Anderson et al., 1992).

We start our consideration from OWB stage appearing during the pulse propagation in the normal dispersive fiber with $N=30$ showing in Fig. $9(\xi=0.065)$. The optical wave breaking comprises following features. Pulse becomes nearly rectangular with relatively sharp leading and trailing edges and oscillations in the pulse edges arise. The strength of oscillations rises with increasing of $N$. The chirp function shows that there is a nearly linear chirp over most of the pulse width; however the steep transition regions are developed at the leading and trailing edges. In the spectrum we can see the development of sidelobes. The appearing of the OWB has been explained by the combined action of SPM and normal GVD (Tomlinson et al., 1985; Anderson et al., 1992). Normal GVD tends to change a nonlinear double-peak chirp pattern induced by SPM. A steepening of the chirp function is occurred, because the red-shifted light near the leading edge travels faster and overtakes the unshifted light in the forward tail of the pulse. The opposite scenario occurs for the blueshifted light near the trailing edge. In both cases, the leading and trailing regions of the pulse contain light at two different frequencies which interfere. Owing to such interference the oscillations near the pulse edges arise. Moreover nonlinear mixing of the overlapped pulse components with different frequencies creates new frequencies. This leads to the development of sidelobes in the spectrum profile.
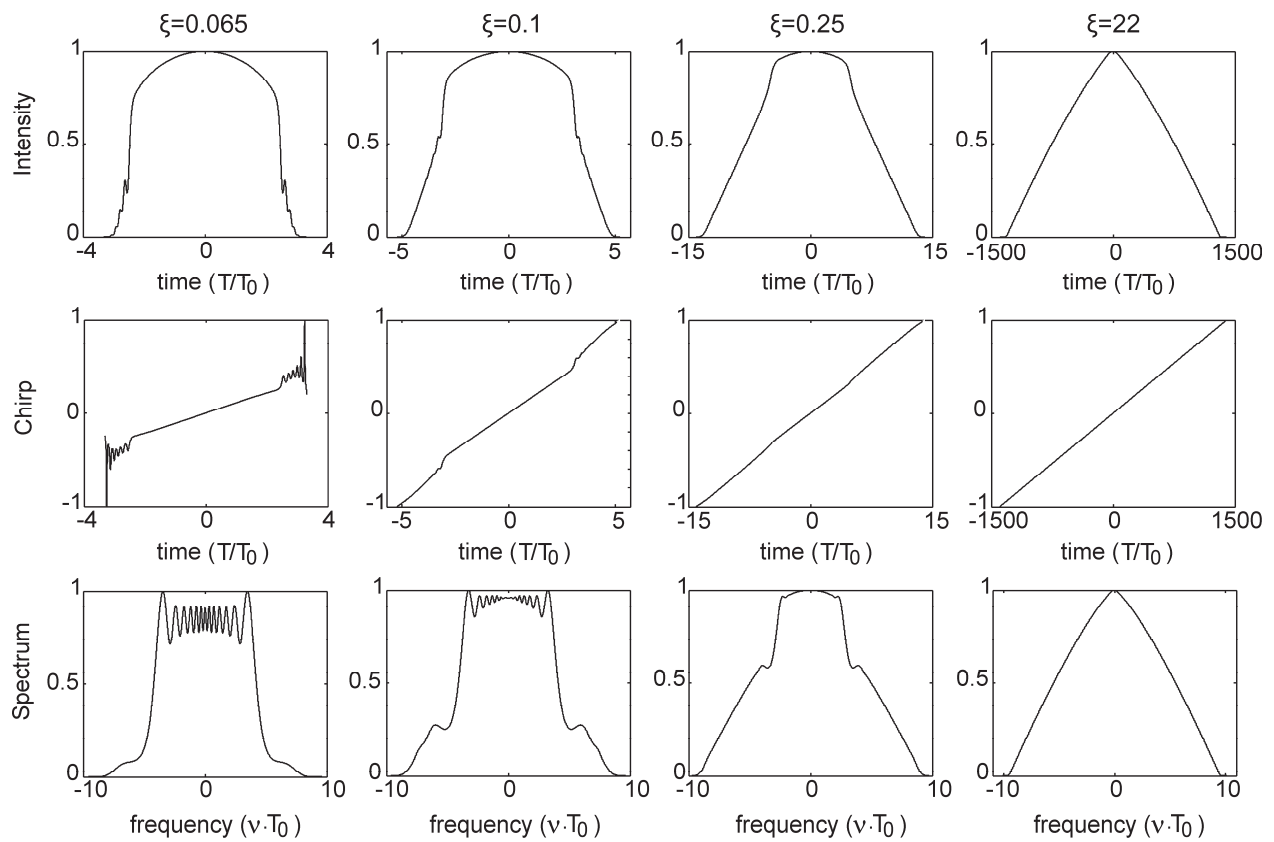

Fig. 9. Evolution of initial Gaussian pulse in a fiber showing optical wave breaking cancellation $(N=30)$. First row comprises pulse temporal intensity over the increasing length $\xi$; second row - instantaneous frequency (chirp); third row - pulse spectrum. All quantities are normalized to their maxima; time and frequency are normalized to the initial pulse width. 
Now let us look on the pulse reshaping at the longer propagation distance. It was shown that oscillations induced by OWB are vanishing at longer distances and pulse transforms to the chirped trapezium-shaped pulse (Karlsson, 1994). Here we investigate this in a more detailed way. One can see from Fig. $9(\xi=0.1$ and $\xi=0.25)$ that oscillations of the chirp function decay at the pulse edges, such that only two bends remain around the pulse center. The magnitudes of these bends decrease with distance, thus actually chirp tends to flattening. At the same time oscillations of the pulse intensity envelope decay as well and we can see also two symmetrical bends on the pulse intensity profile (trapezium-shaped pulse). These bends becomes weaker and moves to the center of the pulse with increasing of propagation distance. A quite long propagation distance is required to complete this process. Only at $\xi=22$ there are no any bends in the pulse intensity profile. The same behavior one can observe in the pulse spectrum, i.e. the bends indicating spectral sidelobes moves to the center of the spectrum and their magnitude decreases. In addition the SPM induced spectral oscillations decay in the top of the spectrum and SPM-induced broadening of the spectrum is stopping. At the last stage $(\xi=22)$ pulse spectrum is smooth also and it actually repeats temporal intensity profile.

Thus, we can see that OWB fully vanishes at the longer propagation distance and pulse temporal shape and spectrum is fully smooth with linear chirp. One should note that the resulted pulse shape is note trapezium, rather triangular. We explain OWB cancellation by the following way. It is clear that the mentioned above picture of OWB onset doesn't freeze with propagation distance. Owing to the normal dispersion a frequency-shifted light should overtake the unshifted light in the pulse edges more and more with propagation distance. When this frequency-shifted light completely overtakes the unshifted light one can observe actually the formation of low-intensity pulse wings both in the temporal and spectral profile (Fig. 9), the chirp function is linear within these wings. The maximum shifted light from initial nonlinear profile overtakes an unshifted light the fastest, however with increasing the propagation distance the less shifted light overtakes it as well. Owing to that low intensity pulse wings expand up to the pulse peak. The frequency difference between the shifted light and unshifted one continuously reduces during this process, therefore optical shock relaxes and a flattening of chirp function occurs. This is accompanied by reducing the bends at the pulse edges and finally we obtain smooth pulse profile with a linear chirp.

So, we can highlight the main trend of the pulse evolution: normal GVD tends to flatten SPM induced nonlinear chirp pattern with propagation distance. At first this leads to the steepening of the chirp function and appearing the OWB, but then with increasing of propagation distance the chirp flattens and OWB vanishes. However, the question remains: How to find the length where OWB fully vanishes and pulse shape is already smooth? It is clear that in this case the pulse shape and spectrum profile should not include any bends. The straightforward way here is to calculate a second derivative of the temporal intensity function or spectral intensity function. Zeros of those second derivatives will indicate the presence of the bend's point. Figure 10 illustrates application of the second derivative for the analysis of the pulse shape. There are shown Gaussian pulse, parabolic pulse and pulse obtained due to nonlinear reshaping of initial Gaussian pulse after the vanishing of OWB. All shown waveforms in Fig. 10 possess only two symmetrical zeroes of the second derivatives. For Gaussian pulse the bends are located near the center of the pulse edges, whereas bends of parabolic pulse are shifted to the foot of the pulse. Due to the sharp 
transitions between zero background and nonzero parabolic profile (resulted from (4)), the appropriate second derivative demonstrates here sharp peaks. In the central part 2-nd derivative of parabolic pulse is horizontal and negative. A pulse obtained due to nonlinear reshaping demonstrates mixed features. Symmetrical bends here are also shifted to the foot of the pulse; however they are not so sharp. In the central part 2-nd derivative is not fully horizontal and moreover a dip in the center remains similar to that one in the Gaussian profile.

Thus, we can see that pulses with smooth edges possess only two symmetrical zeroes indicating the transition between the foot of the pulse and pulse edges. More number of zeroes indicates uneven pulse edges. We can use this in order to characterize the transformation of the pulse shape from the perturbed shape toward the smooth one.
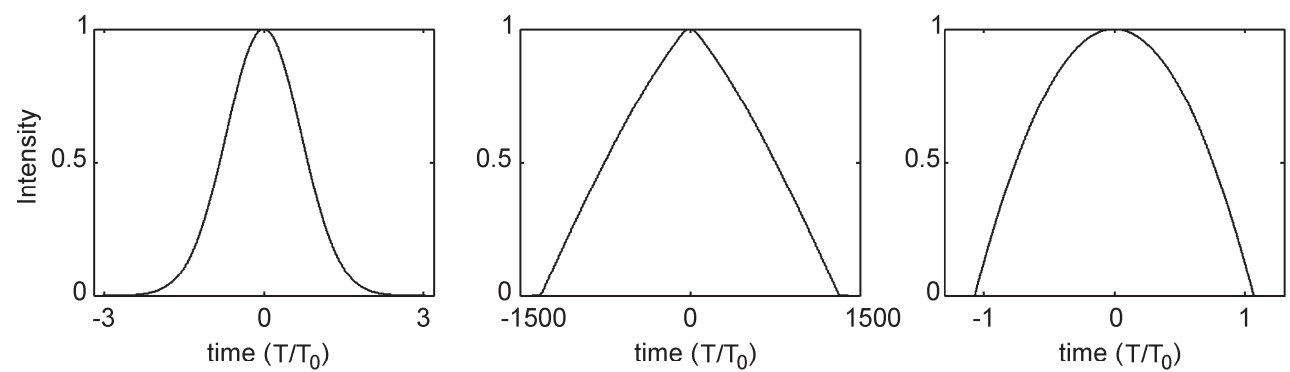

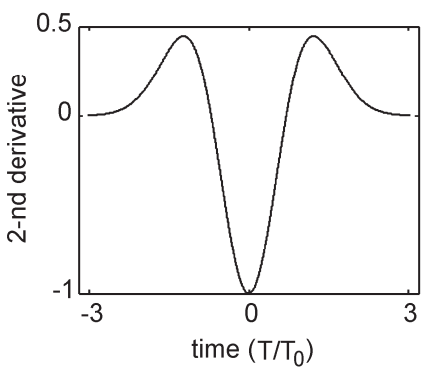

(a)

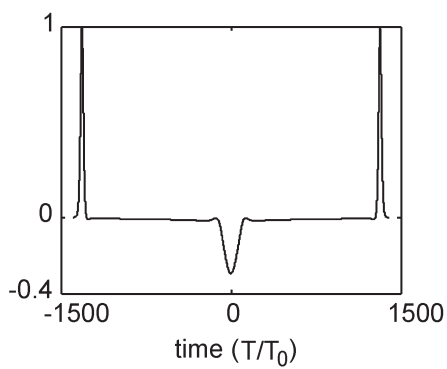

(b)

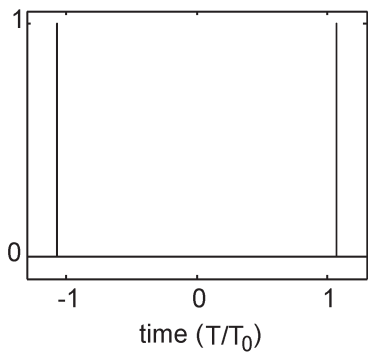

(c)

Fig. 10. Pulse waveforms (top raw) and appropriate 2-nd derivative (bottom row). (a) This column shows Gaussian waveform. (b) This column shows pulse waveform obtained after the optical wave breaking cancelled ( $N=30, \xi=22)$. (c) This column shows parabolic waveform. Pulse temporal intensities are normalized to its maxima; 2-nd derivatives are normalized to its absolute maxima.

Figure 11 shows how the number of zeroes of 2-nd derivative changes during pulse reshaping in a fiber.

At the start point $(\xi=0)$ pulse possesses only 2 zeroes of the second derivatives associated with initial Gaussian profile. However, the number of zeroes increases very fast with pulse propagation and achieves some maximal values indicating pulse shape perturbations due to OWB and SPM induced spectral oscillations. The greater is soliton number $N$, the stronger pulse shape perturbations appear. But after that the number of zeroes decreases and we can see the presence of long horizontal plateau, when the number of zeroes is 6 . At this stage 
pulse waveform is already quite smooth without breaking oscillations or spectral oscillations induced by SPM. There are only two weak symmetrical bends at the pulse edges associated with gradual expansion of the low-intensity temporal and spectral wings. When this process is completed, the number of zeroes of the second derivative goes down to 2 .

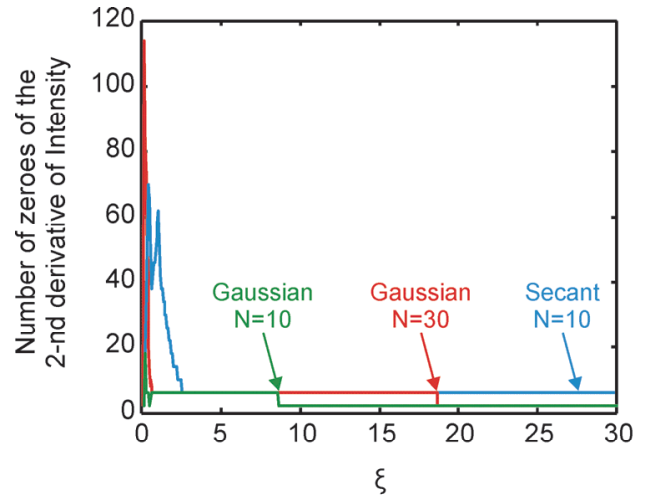

(a)

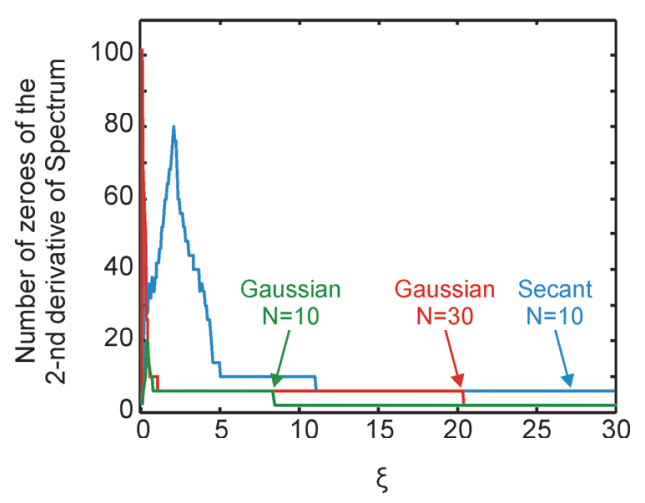

(b)

Fig. 11. (a) Number of zeroes of the second derivative of pulse temporal intensity depending on propagation distance. (b) Number of zeroes of the second derivative of pulse spectrum.

From Fig. 11 we can see that initial Gaussian pulse with $N=10$ becomes smooth the fastest $\xi>9$, here both the temporal intensity and spectrum have smooth profile. For initial Gaussian pulse with $N=30$ it is required longer propagation distance $\xi>21$. In the case of initial secant pulse we can see that bends in the temporal and spectral profile remains until $\xi=30$. This is related to the stronger distortions due to OWB in the case of initial secant pulse.

\subsection{Pulse shape transformations after OWB cancellation}

Here we are interested in the pulse shape transformations in the steady-state regime after OWB cancellation. One can see that the resulted pulse shape here strongly differs (see Fig. 9) from the quasi-parabolic shape achieved in the weak nonlinear case, it is rather triangular. Indeed, recently it has been found that one can achieve a pulse with perfect triangular pulse shape from the initial chirped Gaussian pulse with $N=10$ at $\xi=0.33$ (Boscolo et al., 2008; Wang et al., 2010). Triangular pulses as well as parabolic pulses are highly desired for a range of photonic applications. Possible applications include: add-drop multiplexing (Parmigiani et al., 2009) or doubling of the optical signals (Latkin et al., 2009).

In order to investigate pulse transformations towards triangular pulse shape we introduce the following triangular fitting function:

$$
\left\{\begin{array}{ll}
A_{t}(T)=\sqrt{P_{t}} \sqrt{1-\left|T /\left(T_{t} \sqrt{2.5}\right)\right|}, & |T| \leq T_{t} \sqrt{2.5} \\
A_{t}(T)=0, & |T|>T_{t} \sqrt{2.5}
\end{array},\right.
$$


where $P_{t}$ is the peak power of the triangular pulse, $T_{t}$ is the duration of the triangular pulse (the half-width at 1 /e intensity point), $U_{t}=P_{t} T_{t} \sqrt{2.5}$ is the energy of triangular fit pulse.

Applying (3) and (7) we can investigate pulse transformations towards the triangular shape in the same way it was done above for parabolic pulse shape.

Figure 12 shows transformations of the initial chirped Gaussian pulse with $(N=10, C=-4)$. We can see that temporal spectral and temporal curves achieves minimum near $\xi=0.4$. The triangular misfit parameter here is $M \sim 0.03$, whereas for the spectral shape it is slightly higher. However, we can see from Fig. 12 a) that these minima are very sharp and with increasing the propagation distance the pulse shape and spectrum do not remain triangular.

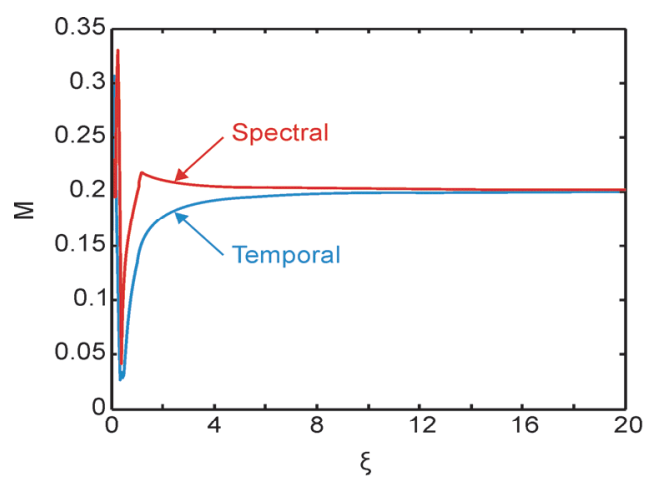

(a)

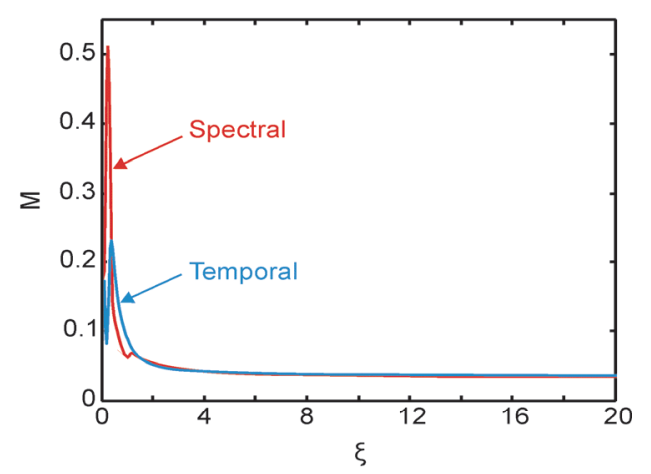

(b)

Fig. 12. a) Evolution of the misfit parameter $M$ versus $\xi$ for the temporal and spectral pulse shapes $(N=10)$. a) - triangular fitting; b) - parabolic fitting.

Fig. 12 b) surprisingly shows that instead of triangular shape we obtain here a fully parabolic pulse in the steady-state regime. Parabolic misfit parameter here is $M<0.04$ from $\xi>6$ both for temporal and spectral profile and moreover, chirp is linear here (Fig. 13).

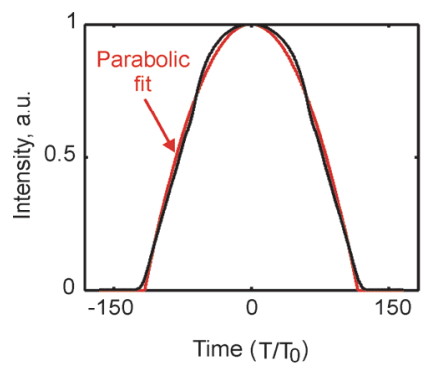

a)

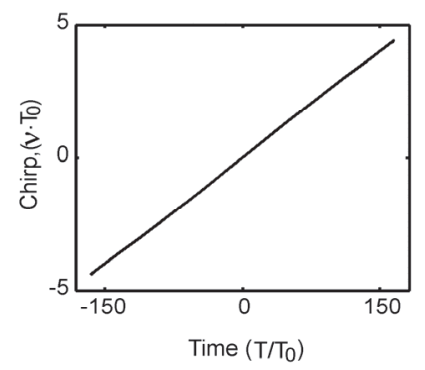

b)

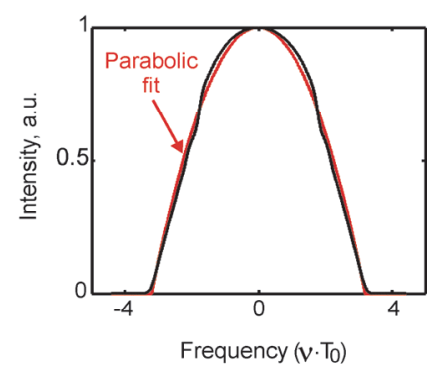

c)

Fig. 13. Normalized pulse temporal intensity $(M=0.039)-(a)$, spectrum $(M=0.038)-(c)$ and chirp (b) in the steady-state regime produced from initial Gaussian pulse with negative chirp $(N=10, \xi=6, C=-4)$. Red curves show corresponding parabolic fits. 
Thus, in the steady-state regime we can obtain parabolic pulses not only in the weak nonlinear case $(1.5<N<2.5)$, but also at the higher pulse powers.

In spite of that it is also possible to obtain triangular pulses as well in the steady-state regime. Figure 14 a) clearly shows that applying initial secant pulse without any chirp we can obtain triangular pulses in the steady-state regime. One can see that triangular misfit parameter is $M<0.04$ from $\xi>10$ both for temporal and spectral profile. In contrast to that Fig. 14 b) shows that in the case of initial Gaussian pulse the deviation from the triangular fit is sufficiently higher.

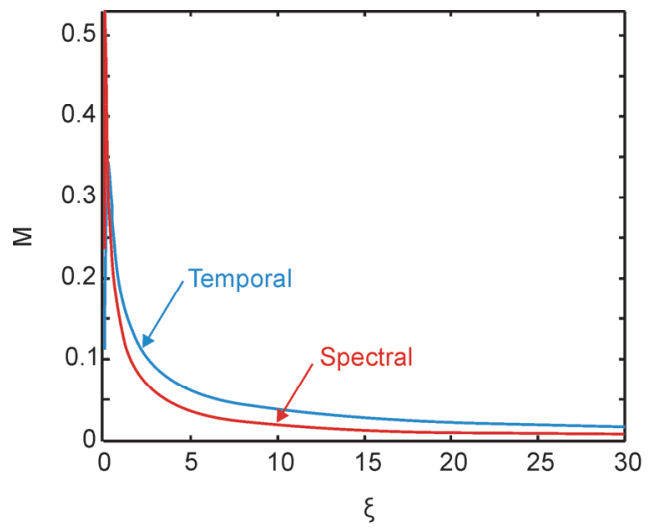

(a)

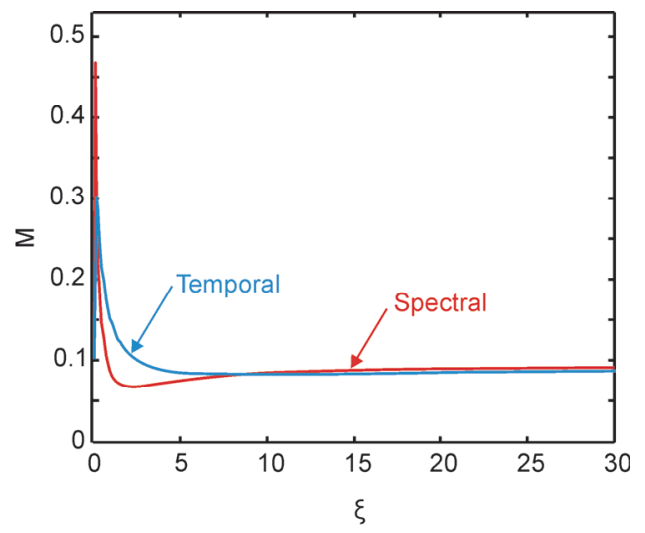

(b)

Fig. 14. a) Evolution of the triangular misfit parameter $M$ versus $\xi(N=10)$.a) - initial secant pulse; b) - initial Gaussian pulse.

Figure 15 shows the resulted pulse shape in the steady-state regime obtained from the initial unchirped secant pulse. Pulse shape and spectrum are fully triangular over the whole pulse with the exception of a small flat top. The chirp is also linear here.

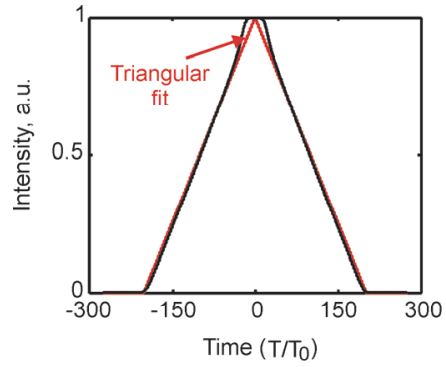

a)

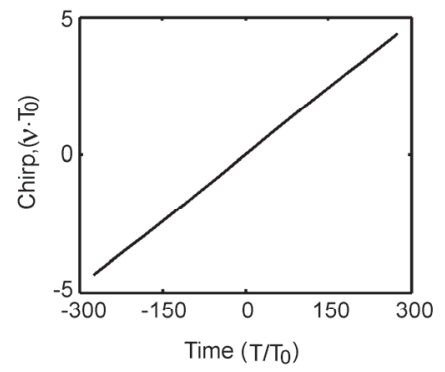

b)

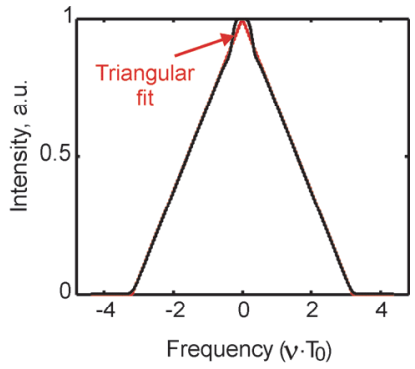

c)

Fig. 15. Normalized pulse temporal intensity $(M=0.037)-(a)$, spectrum $(M=0.018)-(c)$ and chirp (b) in the steady-state regime produced from initial secant pulse $(N=10, \xi=10)$. Red curves show corresponding triangular fits. 


\section{Conclusion}

We have investigated pulse shape transformations in passive optical fibers in the steadystate regime. In the case of super-Gaussian pulse shape (2-nd order) the resulted pulse waveform tends to the fully parabolic very fast. In the case of initial Gaussian pulse quasiparabolic pulses $(M \sim 0.07)$ with linear chirp can be obtained in the weak nonlinear case at fiber length $\xi>5$. In the case of initial secant pulse the steady-state regime arises at much longer propagation distance and obtained pulse shape differs stronger from the parabolic one. The value of soliton order $N$ strongly affects on the pulse shape in the steady-state. When soliton order lies within the following range $1.5<N<2.5$, the shape of quasiparabolic pulse is closest to the parabolic one. Application of positively chirped pulses is preferable to achieve quasi-parabolic pulses with minimal deviation from the parabolic profile both for temporal and spectral shapes at the shortest propagation distance. Thirdorder dispersion is able to affect strongly on the pulse shape in the steady-state regime leading to the pulse distortions due to optical shock-type instabilities. It was found that the dispersion length's ratio $L_{D}^{\prime} / L_{D}>30$ provides undistorted pulse shape. The presence of losses in the fiber damps the strength of pulse reshaping and the resulted pulse shape differs stronger from the parabolic profile, whereas gain enhances pulse reshaping towards the parabolic shape. In the strong nonlinear case $(N \geq 10)$ it is also possible to achieve parabolic pulses in the steady-state regime from the initially chirped Gaussian pulses. Moreover, one can obtain also triangular pulses from the unchirped secant pulses.

\section{References}

Agrawal, G. P. (2007). Nonlinear Fiber Optics. Academic Press, Boston.

Akhmediev, N. \& Ankiewicz, A. (Ed(s).). (2008). Dissipative Solitons: From optics to biology and medicine, Springer, Berlin-Heidelberg.

Anderson, D.; Desaix, M.; Lisak, M. \& Quiroga-Teixeiro, M. L. (1992). Wave breaking in nonlinear-optical fibers. J. Opt. Soc. Am. B, Vol. 9, No. 8, pp. 1358-1361.

Anderson, D.; Desaix, M.; Karlsson, M.; Lisak, M. \& Quiroga-Teixeiro, M. L. (1993). Wavebreaking-free pulses in nonlinear-optical fibers. J. Opt. Soc. Am. B, Vol. 10, No. 7, pp. 1185-1190.

Bale, B. G. \& Boscolo, S. (2010). Impact of third-order fibre dispersion on the evolution of parabolic optical pulses. J. Opt., Vol. 12, No. 1, pp. 015202.

Barenblatt, G. I. (1996). Scaling, Self-Similarity, and Intermediate Asymptotics. Cambridge University Press, Cambridge.

Boscolo, S.; Latkin, A. I. \& Turitsyn, S. K. (2008). Passive Nonlinear Pulse Shaping in Normally Dispersive Fiber Systems, IEEE J. Quantum Electron., Vol. 44, No. 12, pp. 1196-1203.

Brabec, T.; Spielmann, Ch. \& Krausz, E. (1991). Mode locking in solitary lasers. Opt. Lett., Vol. 16, No. 24, pp. 1961-1963.

Dudley, J. M.; Genty, G.; Coen, S. (2006). Supercontinuum generation in photonic crystal fiber. Rev. Mod. Phys., Vol. 78, No. 4, pp. 1135-1184.

Dudley, J. M.; Finot, C.; Richardson, D. J. \& Millot, G. (2007). Self-similarity in ultrafast nonlinear optics. Nature Physics, Vol. 3, pp. 597-603. 
Duling III, I. N. (1991). Subpicosecond all-fibre erbium laser. Electron. Lett., Vol. 27, No. 6, pp. 544-545.

Fermann, M. E.; Kruglov, V. I.; Thomsen, B. C.; Dudley, J. M. \& Harvey, J. D. (2000). Selfsimilar propagation and amplification of parabolic pulses in optical fibers. Phys. Rev. Lett., Vol. 84, No. 26, pp. 6010-6013.

Finot, C.; Parmigiani, F.; Petropoulos, P. \& Richardson, D. J. (2006). Parabolic pulse evolution in normally dispersive fiber amplifiers preceding the similariton formation regime. Opt. Express, Vol. 14, No. 8, pp. 3161-3170.

Finot, C.; Provost, L.; Petropoulos, P. \& Richardson, D. J. (2007). Parabolic pulse generation through passive nonlinear pulse reshaping in a normally dispersive two segment fiber device," Opt. Express, Vol. 15, No. 3, pp. 852-864.

Finot, C.; Dudley, J. M.; Kibler, B.; Richardson, D. J.; Millot, G. (2009). Optical Parabolic Pulse Generation and Applications. IEEE J. Quantum Electron., Vol. 45, No. 11, pp. $1482-1489$.

French, W. G.; MacChesney, J. B.; O'Connor, P. D.; Tasker, G. W. (1974). Optical waveguides with very low losses, Bell Syst. Tech. J., Vol. 53, No. 5, pp. 951-954.

$\mathrm{Gu}$, C.H. (Ed(s).). (1995). Soliton Theory and its Applications, Springer, New York.

Hasegawa, A. \& Matsumoto, M. (2003). Optical Solitons in Fibers (3rd ed.), Springer-Verlag, Berlin.

Hasegawa, A. \& Tappert, F. (1973). Transmission of stationary nonlinear optical pulses in dispersive dielectric fibers. I. Anomalous dispersion. Appl. Phys. Lett., Vol. 23, No.3, pp. 142-144.

Hirooka, T. \& Nakazawa, M. (2004). Parabolic pulse generation by use of a dispersiondecreasing fiber with normal group-velocity dispersion," Opt. Lett., Vol. 29, No. 5, pp. $498-500$.

Ilday, F. Ö.; Buckley, J. R.; Clark, W. G. \& Wise, F. W. (2004). Self-similar evolution of parabolic pulses in a laser. Phys. Rev. Lett., Vol. 92, No. 21, pp. 213902.

Ippen, E. P. \& Stolen, R. H. (1972). Stimulated Brillouin scattering in optical fibers. Appl. Phys. Lett., Vol. 21, No. 11, pp. 539-541.

Kao, K. C.; Hockham, G. A. (1966). Dielectric-fibre surface waveguides for optical frequencies. Proc. IEE, Vol. 113, No. 7, pp. 1151-1158.

Karlsson, M. (1994). Optical fiber-grating compressors utilizing long fibers. Opt. Comm., Vol. 112, No. 1-2, pp. 48-54.

Kivshar, Y. S. \& Agrawal, G. P. (2003). Optical solitons From Fibers to Photonic Crystals. Academic Press, San Diego.

Kodama, Y. and Hasegawa, A. (1987). Nonlinear pulse propagation in a monomode dielectric guide. IEEE J. Quantum Electron., Vol. 23, No. 5, pp. 510-524.

Kruglov, V. I.; Peacock, A. C.; Harvey, J. D. \& Dudley, J. M. (2002). Self-similar propagation of parabolic pulses in normal dispersion fiber amplifiers. J. Opt. Soc. Am. B, Vol. 19, No. 3, pp. 461-469.

Kruglov, V. I., Peacock, A. C. \& Harvey, J. D. (2003). Exact self-similar solutions of the generalized nonlinear Schrdinger equation with distributed coefficients, Phys. Rev. Lett., Vol. 90, No. 11, pp. 113902.

Latkin, A. I.; Turitsyn, S. K. \& Sysoliatin, A. A. (2007). Theory of parabolic pulse generation in tapered fiber. Opt. Lett., Vol. 32, No. 4, pp. 331-333. 
Latkin, A. I.; Boscolo, S.; Bhamber, R. S. \& Turitsyn, S. K. (2009) Doubling of optical signals using triangular pulses. J. Opt. Soc. Am. B, Vol. 26, No. 8, pp. 1492-1496.

Miya, T.; Terunuma, Y.; Hosaka, T.; Miyashita, T. (1979). Ultimate low-loss single mode fiber at $1.55 \mu \mathrm{m}$. Electron. Lett., Vol. 15, No. 4, pp. 106-108.

Mollenauer, L. F.; Stolen, R. H.; \& Gordon, J. P. (1980). Experimental Observation of Picosecond Pulse Narrowing and Solitons in Optical Fibers. Phys. Rev. Lett., Vol. 45, No. 13, pp. 1095-1098.

Parmigiani, F.; Finot, C.; Mukasa, K.; Ibsen, M.; Roelens, M. A.; Petropoulos, P. \& Richardson, D. J. (2006). Ultra-flat SPM-broadened spectra in a highly nonlinear fiber using parabolic pulses formed in a fiber Bragg grating. Opt. Express, Vol. 14, No. 17, pp. 7617-7622.

Parmigiani, F.; Petropoulos, P.; Ibsen, M.; Almeida, P. J.; Ng, T. T. \& Richardson, D. J. (2009) Time domain add-drop multiplexing scheme enhanced using a saw-tooth pulse shaper Opt. Express, Vol. 17, No. 10, pp. 8362-8369.

Rothenberg, J. E. (1989). Femtosecond optical shocks and wave breaking in fiber propagation. J. Opt. Soc. Am. B, Vol. 6, No. 12, pp. 2392-2401.

Schreiber, T.; Nielsen, C. K.; Ortac, B.; Limpert, J. P. \& Tünnermann, A. (2006). Microjoulelevel all-polarization-maintaining femtosecond fiber source. Opt. Lett., Vol. 31, No. 5, pp. 574-576.

Stolen, R. H. \& Askin, A. (1973). Optical Kerr effect in glass waveguide. Appl. Phys. Lett., Vol. 22, No.6, pp. 294-296.

Stolen, R. H.; Bjorkholm, J. E. \& Ashkin, A. (1974). Phase-matched three-wave mixing in silica fiber optical waveguides. Appl. Phys. Lett., Vol. 24, No. 7, pp. 308-310.

Stolen, R. H. \& Lin, C. (1978). Self-phase-modulation in silica optical fibers. Phys. Rev. A, Vol. 17, No. 4, pp. 1448-1453.

Sukhoivanov, I. A.; Iakushev, S. O.; Petrov, S. I.; Shulika, O. V. Optical wave breaking cancellation in the far dispersion field of optical fiber, Proceedings of Photonics Society Summer Topical Meeting Series, pp. 86-87, Playa del Carmen, Mexico, July 19-21, 2010.

Tamura, K. \& Nakazawa, M. (1996). Pulse compression by nonlinear pulse evolution with reduced optical wave breaking in erbium-doped fiber amplifiers. Opt. Lett., Vol. 21, No. 1, pp. 68-70.

Tomlinson, W. J.; Stolen, R. H. \& Johnson, (1985). A. M. Optical wave breaking of pulses in nonlinear optical fibers. Opt. Lett., Vol. 10, No. 9, pp. 457-459.

Wang, H.; Latkin, A. I.; Boscolo, S.; Harper, P. \& Turitsyn, S. K. (2010). Generation of triangular-shaped optical pulses in normally dispersive fibre. J. Opt., Vol. 12, No. 3, pp. 035205.

Yakushev, S. O.; Shulika, O. V.; Sukhoivanov, I. A.; Andrade-Lucio, J. A.; Garcia-Perez, A. Quasi-Parabolic Pulses in the Far Field of Dispersion of Nonlinear Fiber, Proceedings of Frontiers in Optics/Laser Science, pp. FTuR2, Rochester, NY, USA, October 24-28, 2010.

Zakharov, V. E. \& Shabat, A. B. (1972). Exact theory of two-dimensional self-focusing and one-dimensional self-modulation of waves in nonlinear media. Sov. Phys. JETP, Vol. 34, pp. 62-69. 
Zeytunyan, A.; Yesayan, G.; Mouradian, L.; Kockaert, P.; Emplit, P.; Louradour, F.; Barthélémy, A. (2009). Nonlinear-dispersive similariton of passive fiber. J. Europ. Opt. Soc. Rap. Public., Vol. 4, pp. 09009.

Zeytunyan, A. S.; Khachikyan, T. J.; Palandjan, K. A.; Esayan, G. L. \& Muradyan, L. Kh. (2010). Nonlinear dispersive similariton: spectral interferometric study. Quantum Electron., Vol. 40, No. 4, pp. 327. 


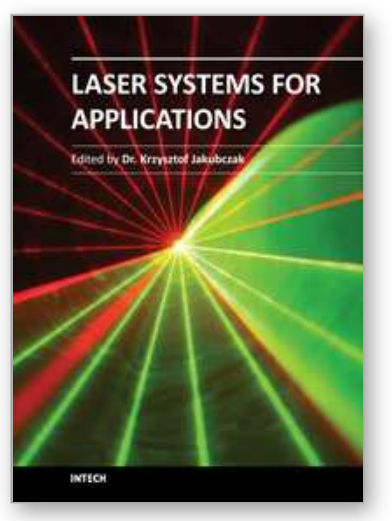

\author{
Laser Systems for Applications \\ Edited by Dr Krzysztof Jakubczak
}

ISBN 978-953-307-429-0

Hard cover, 308 pages

Publisher InTech

Published online 14, December, 2011

Published in print edition December, 2011

This book addresses topics related to various laser systems intended for the applications in science and various industries. Some of them are very recent achievements in laser physics (e.g. laser pulse cleaning), while others face their renaissance in industrial applications (e.g. $\mathrm{CO} 2$ lasers). This book has been divided into four different sections: (1) Laser and terahertz sources, (2) Laser beam manipulation, (3) Intense pulse propagation phenomena, and (4) Metrology. The book addresses such topics like: Q-switching, mode-locking, various laser systems, terahertz source driven by lasers, micro-lasers, fiber lasers, pulse and beam shaping techniques, pulse contrast metrology, and improvement techniques. This book is a great starting point for newcomers to laser physics.

\title{
How to reference
}

In order to correctly reference this scholarly work, feel free to copy and paste the following:

S. O. lakushev, I. A. Sukhoivanov, O. V. Shulika, J. A. Andrade-Lucio and A.G. Perez (2011). Nonlinear Pulse Reshaping in Optical Fibers, Laser Systems for Applications, Dr Krzysztof Jakubczak (Ed.), ISBN: 978-953307-429-0, InTech, Available from: http://www.intechopen.com/books/laser-systems-for-applications/nonlinearpulse-reshaping-in-optical-fibers

\section{INTECH}

open science | open minds

\section{InTech Europe}

University Campus STeP Ri

Slavka Krautzeka 83/A

51000 Rijeka, Croatia

Phone: +385 (51) 770447

Fax: +385 (51) 686166

www.intechopen.com

\section{InTech China}

Unit 405, Office Block, Hotel Equatorial Shanghai

No.65, Yan An Road (West), Shanghai, 200040, China

中国上海市延安西路65号上海国际贵都大饭店办公楼 405 单元

Phone: +86-21-62489820

Fax: +86-21-62489821 
(C) 2011 The Author(s). Licensee IntechOpen. This is an open access article distributed under the terms of the Creative Commons Attribution 3.0 License, which permits unrestricted use, distribution, and reproduction in any medium, provided the original work is properly cited. 